\title{
Computer simulation of deformation and fracture of small crystals by molecular dynamics method
}

\author{
MASAO DOYAMA \\ The Nishi-Tokyo University, Uenohara, Yamanashi 409-01, Japan
}

\begin{abstract}
Body-centred cubic iron whiskers having [100] and [110] axes were pulled in a molecular dynamics simulation using a supercomputer. The upper yield stress close to the theoretical strength was found. Above the upper yield stress, phase transformation was observed; at the same time the stress was greatly reduced. A new possible mechanism of twinning is proposed. The whiskers were pulled until they had broken into two pieces. Copper small crystals with and without a notch were sheared. It was observed that the edge dislocations were created at the surface and moved through and escaped from the crystals. Copper small single crystals with a notch were pulled. A half-dislocation was created near the tip of the notch. Sharp yield stress was observed. In medium deformation dislocations on different slip planes were created. Due to the cutting of dislocations the tensile stress increased.
\end{abstract}

Keywords. Computer simulation; deformation; fracture; small crystals; molecular dynamics method.

\section{Introduction}

Plastic deformation of metals is caused by the creation and motion of dislocations. Fracture is a very important problem in engineering and science but atomistic explanations of it have not been verified. Molecular dynamics has found much application in materials science and engineering. It is quite useful for simulating the creation and motion of dislocations during plastic deformation in metals. It is also useful for simulating deformation until the specimen is fractured.

\section{Interatomic potentials}

The interaction potentials between the $i$ th and $j$ th atoms are often represented by a pairwise potential. The simplest pair potential is only a function of the distance between two atoms. In this paper, the two-body interaction between two atoms in body-centred iron is represented by

$$
\Phi\left(r_{i j}\right)=a_{1}\left(r_{i j}-a_{2}\right)^{4}+a_{3}\left(r_{i j}-a_{4}\right)^{2}+a_{5},
$$

where $a_{1}=-0.188917 \mathrm{eV} / \AA^{4}, a_{2}=1.82709 \AA, a_{3}=1 \cdot 70192 \mathrm{eV} / \AA^{2}, a_{4}=2.50849 \AA$, $a_{5}=0.198294 \mathrm{eV}$, and $r_{i j}$ is the distance between the $i$ th and $j$ th atoms. This potential was determined by Pak and Doyama (1969). It has been used for calculating point defects in iron, the structure of amorphous iron, etc. It is smoothly truncated at $3.44 \AA$ between the second nearest neighbour and the third nearest neighbour. By this potential body-centred cubic lattice is the most stable configuration. This potential is called $\mathrm{Fe}-1$ potential hereafter. In figure $1 \mathrm{Fe}-1$ is plotted against the distance.

The two-body potential in copper used here (Doyama and Yamamoto 1986) is

$$
\Phi\left(r_{i j}\right)=-2 \cdot 093936\left(r_{i j}-3 \cdot 139365\right)^{2}\left(r_{i j}-2 \cdot 251664\right)(\mathrm{eV}) .
$$




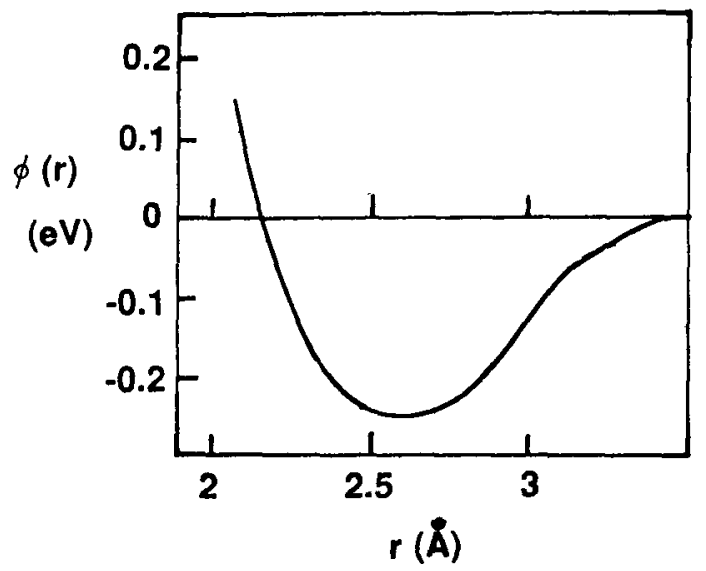

Figure 1. The effective interaction potential between atoms in iron.

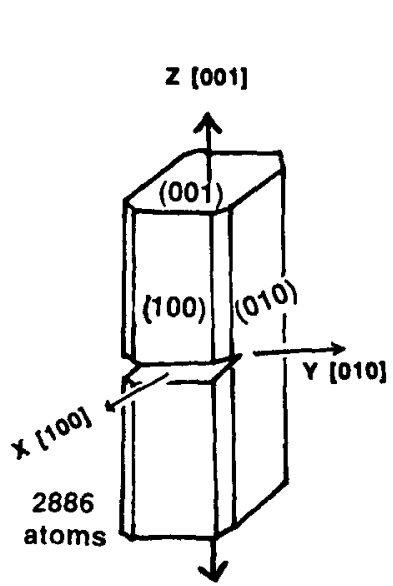

(a)

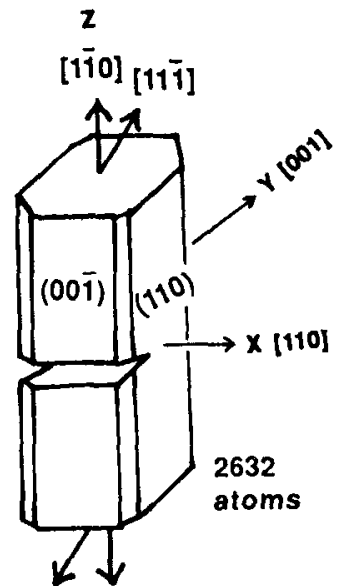

(b)

Figure 2. Iron specimens (a) Fe-1 and (b) Fe-2.

This potential is spherically symmetric and smoothly truncated. It is called potential $\mathrm{Cu}-1$ hereafter. $r_{i}$ is represented in $\AA$.

The interatomic potentials in metals, however, cannot be expressed by two-body interaction. $N$-body embedded function determined by $O h$ and Johnson $(1988,1989)$ is used for copper. This is called potential $\mathrm{Cu}-2$ hereafter.

$$
\begin{aligned}
E_{\text {total }} & =\Sigma E_{i} \\
r_{i j} & =\left|r_{i}-r_{j}\right| \\
E_{i} & =F\left(\rho_{i}\right)+(1 / 2) \Sigma \Phi\left(r_{i j}\right) \\
F(\rho) & =a\left(\rho / \rho_{e}\right)^{n}+b\left(\rho / \rho_{e}\right) \\
\rho_{i} & =\Sigma f\left(r_{i j}\right) .
\end{aligned}
$$


Here $E_{\text {total }}$ is the total internal energy, $E_{i}$ the internal energy associated with atom $i, \rho_{i}$ the electron density at atom $i$ due to all other atoms, $F\left(\rho_{i}\right)$ the embedding energy of the atom into the electron density $\rho_{i}, \Phi\left(r_{i j}\right)$ the two-body central potential between atoms $i$ and $j$ separated by $r_{i j}$ and $f\left(r_{i j}\right)$ the contribution to the electron density at atom $i$ due to atom $j$ at the distance $r_{i j}$ from atom $i$.

$$
\begin{aligned}
& f(r)=f_{\text {old }}(r)-f_{\mathrm{c}}(r) \\
& f_{\mathrm{old}}=f_{\mathrm{e}} \exp \left\{-\beta\left(r / r_{\mathrm{e}}\right)-1\right\} \\
& f_{\mathrm{e}}(r)=f_{\text {old }}\left(r_{\mathrm{c}}\right)+g(r) f_{\mathrm{old}}^{\prime}\left(r_{\mathrm{c}}\right) / g^{\prime}\left(r_{\mathrm{c}}\right) \\
& \Phi(r)=\Phi_{\text {old }}(r)-\Phi_{\mathrm{c}}(r)
\end{aligned}
$$

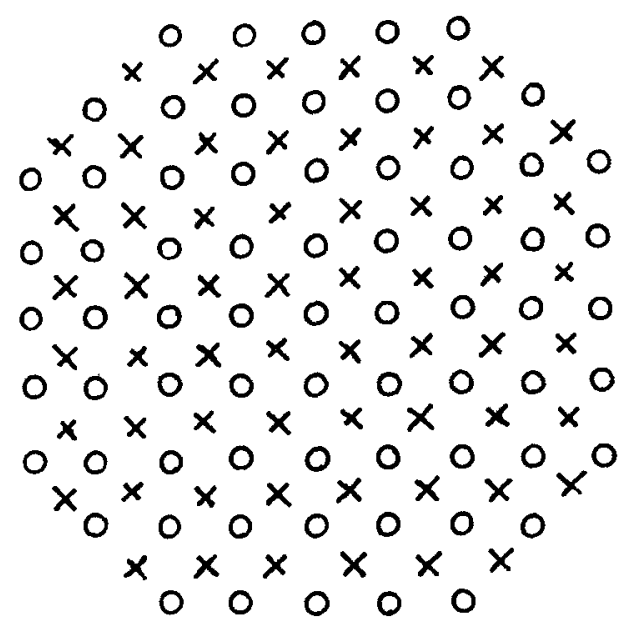

Figure 3. Cross-section of specimen $\mathrm{Fe}-1$.

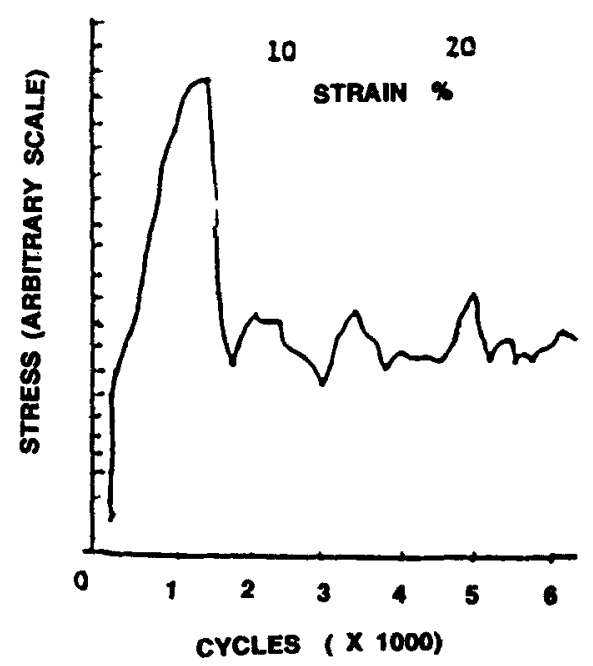

Figure 4. Stress-strain curve of specimen Fe-1. 


$$
\begin{aligned}
& \Phi_{\text {old }}(r)=\Phi_{\mathrm{e}} \exp \left\{-\gamma\left(r / r_{\mathrm{e}}-1\right)\right\} \\
& \Phi_{\mathrm{c}}(r)=\Phi_{\text {old }}\left(r_{\mathrm{c}}\right)+g(r) \Phi_{\text {old }}^{\prime}\left(r_{\mathrm{c}}\right) / g^{\prime}\left(r_{\mathrm{c}}\right) \\
& g(r)=1-\exp \left\{\delta\left(r / r_{\mathrm{c}}\right)-r_{\mathrm{c}} / r_{\mathrm{e}}\right) .
\end{aligned}
$$

For copper Oh and Johnson $(1988,1989)$ give $\beta=5, \gamma=8.5, \delta=20, r_{\mathrm{c}}=1.9 r_{\mathrm{e}}$, $\Phi_{\mathrm{c}}(r)=0.36952 \mathrm{eV}, a=-4.0956, b=-1.6979, n=0.44217$ and $\rho_{\mathrm{e}}=12.793$.

\section{Iron whiskers}

\subsection{Tensile deformation of iron whiskers}

Needle-shaped iron whiskers were pulled in a molecular dynamics simulation in a Hitachi S-810 supercomputer. Two body centred cubic iron specimens were created. Specimen Fe-1 contains 2886 atoms. The axis was [001] direction. The specimen size is $8 a \times 8 a \times 14 a$, where $a$ is the lattice parameter, and is truncated at the edges

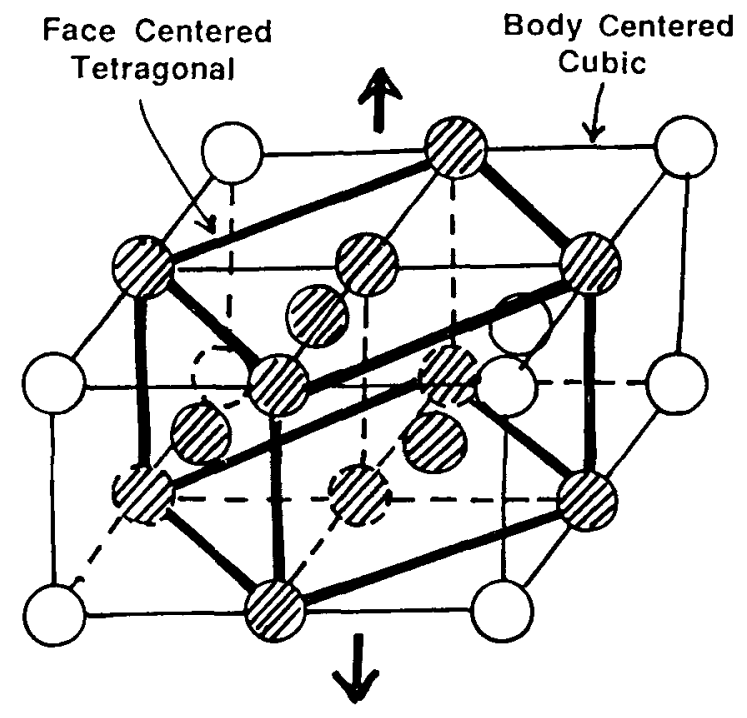

Figure 5. Face-centred tetragonal lattice in a body-centred cubic lattice.

Figure 6. Atomic positions, projected to (010) in specimen Fe-1 at $24.4 \%$ elongation (6200 cycles). 


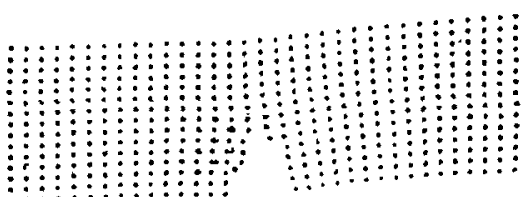

(a)

(c)

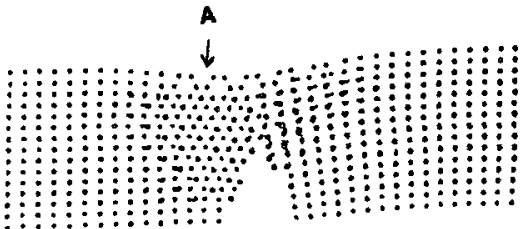

(b)

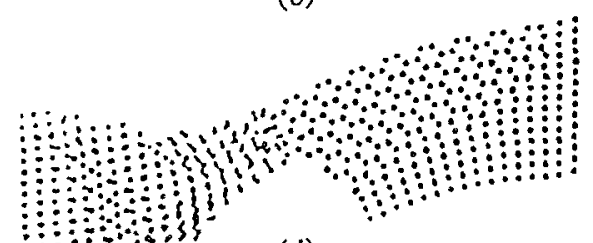

(d)
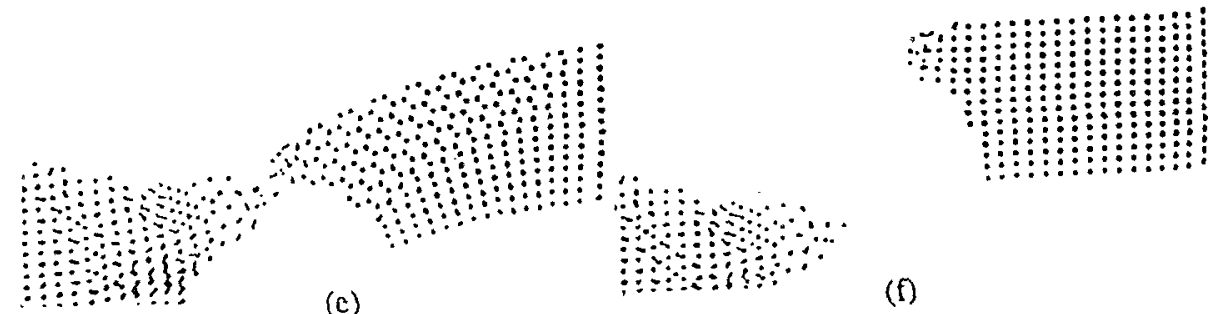

(f)

Figure 7. (a) Atomic positions of specimen $\mathrm{Fe}-2$ at $6.8 \%$ elongation, pulled in [111] direction; (b) in region $A$ twin was initiated; (c) necking was observed at $22 \%$ elongation; (d-f) Fe-2 was completely broken into two pieces.

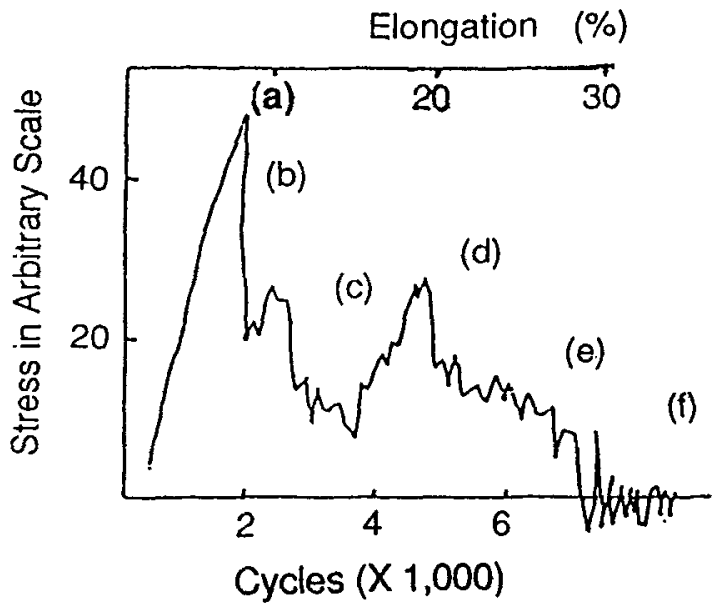

Figure 8. Stress-strain curve for specimen Fe-2 pulled in [111] direction. The letters represent figures of atomic configuration in figure 7 .

(figure 2a). The surfaces are (100), (100), (010), (010), (001) and (001). $x, y$ and $z$ axes are taken in [100], [010] and [001] directions, respectively. The cross-section is plotted in

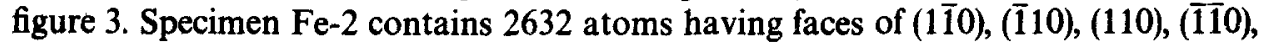
(001) and (001) (figure 2b). 
The iron specimens were first relaxed for 100 cycles by the molecular dynamıs method. In each cycle all the atoms in the specimen are moved toward the resultant forces by neighbouring atoms proportional to the forces. The atoms in two atomic layers at both ends, "holder" regions, were specially treated, extended and held $z$ coordinates. The $z$ direction was taken to be the direction of the whisker axes. Uniform tensile deformation of $0.004(0.4 \%)$ was given in a tensile direction (nominal elongation), then all atoms other than holder regions were relaxed. After each uniform tension was given, atoms were relaxed for 100 cycles, then uniform tension was again given. One hundred cycles of relaxation were performed. This procedure was repeated. The amount of tension each time was $0.004(0.4 \%)$. The atoms in the holder were relaxed in the $x$ and $y$ directions only, not in $z$ direction.

The total force in the $z$ direction of all the atoms in the "holder" region was calculated. This can be taken as the tensile stress. The tensile stress-strain curve is plotted in figure 4 . As seen from the stress-strain curve, very sharp yield stress was observed. Up to the yield stress, the specimen was almost uniformly deformed. Above the yield stress, stress was greatly reduced. At the same time, a face-centred tetragonal lattice was formed. The relationship between the tetragonal face-centred lattice and the body-centred lattice is as follows: $(001)_{\mathrm{bcc}} / /(001)_{\mathrm{fcc}},[110]_{\mathrm{fcc}} / /[100]_{\mathrm{bcc}}$ and $[110]_{\mathrm{fcc}} / /$ $[010]_{\text {bcc }}$. A face-centred tetragonal structure can be considered in a body-centred cubic lattice as shown in figure 5. Applying a tension in the $c$ direction, the $c$ axis is expanded and the $a$ axes are shrunk, then finally face centred cubic structure is obtained. Figure 6 is the projection of atom positions to $x z$ direction at $24 \cdot 4 \%$ elongation.

Specimen Fe-2 was pulled in [11i] direction. In this specimen, the resolved shear stress to [11 1 ] on (111) plane is the highest for one of the slip systems. An easier glide is expected. The slip started at the corner of the tip of the crack, i.e. the place where the crack ends at the surface. Slip started from the tip of the notch. Up to $6.8 \%$ elongation, the specimen was very uniformly extended except very near the crack (figure $7 \mathrm{a}$ ). Above $6.8 \%$,

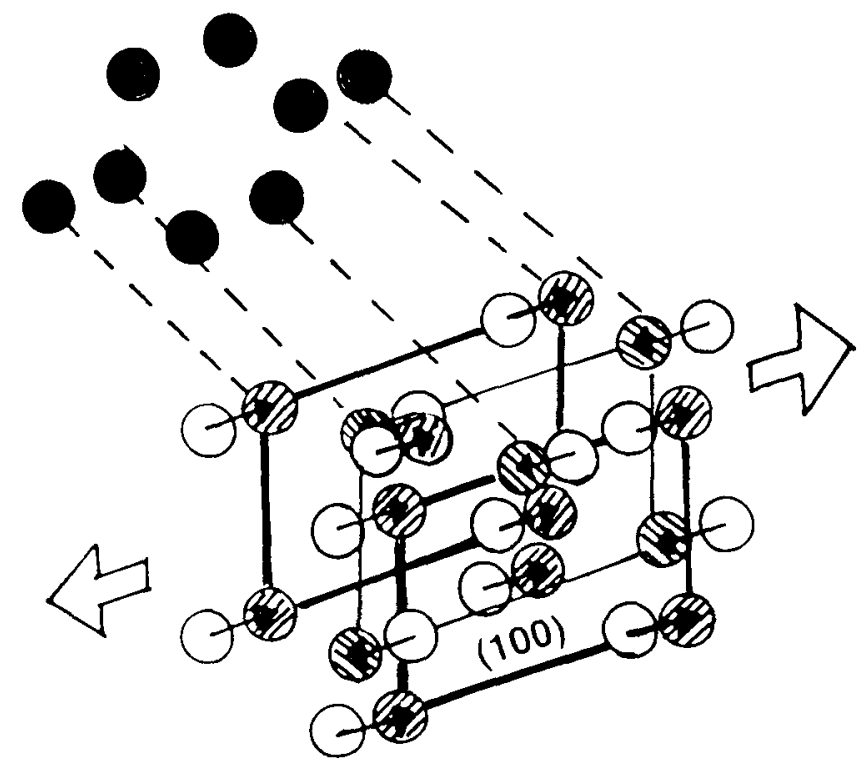

Figure 9. Formation of six-fold symmetry by shuffing (100) planes. 


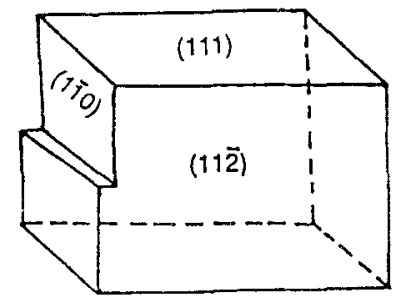

(a)

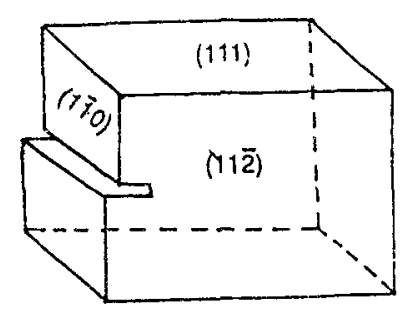

(b)

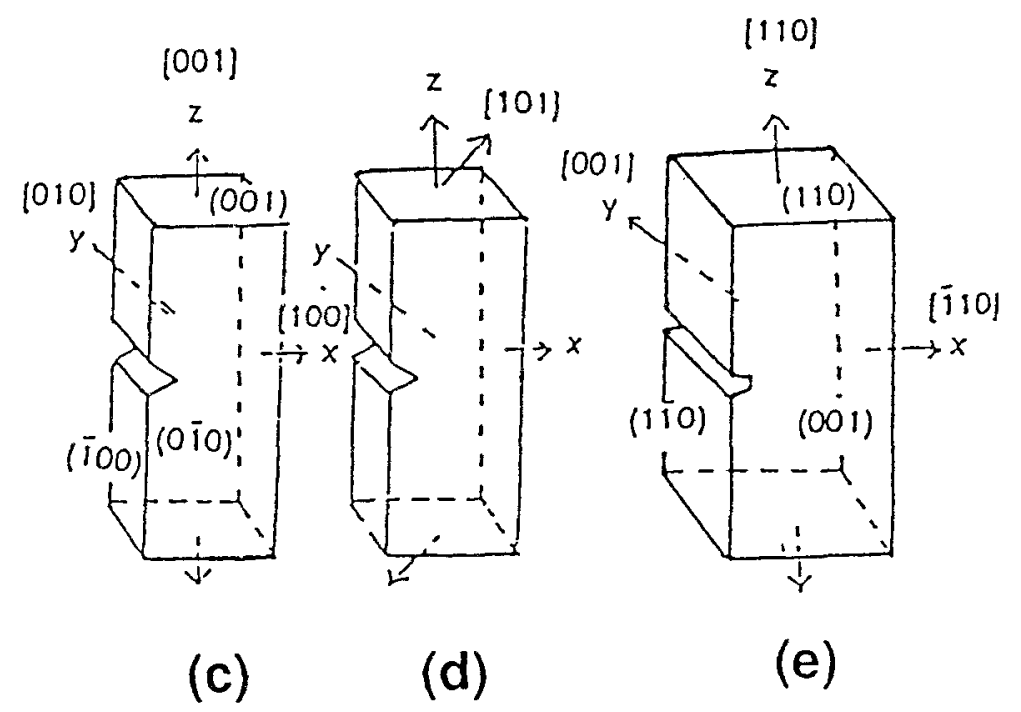

Figure 10. Copper specimens (a) $\mathrm{Cu}-1$, (b) $\mathrm{Cu}-2$, (c) $\mathrm{Cu}-3$, (d) $\mathrm{Cu}-4$ and (e) $\mathrm{Cu}-5$.

the specimen was suddenly transformed. Inside the crystal six-fold symmetrical configuration was observed. This intermediate six-fold symmetry configuration suddenly changed to a twin at about $10 \%$ elongation. Twin was formed in region $\mathrm{A}$ shown in figure $7 \mathrm{~b}$. The twin region expanded as the deformation progressed. The six-fold symmetry region also expanded. At about $17 \%$ elongation, very severe deformation was observed.

The "necking" started above $22 \%$ elongation (figure 7 c). At $28 \%$ elongation, the specimen was broken into two pieces (figure 7d). After fracture, the region of the six-fold symmetry configuration decreased under no stress and the original bodycentred cubic lattice of alpha-iron was obtained (figure $7 \mathrm{~d}$ ).

For specimen $\mathrm{Fe}-2$, the stress-strain curve is shown in figure 8. After the yield point, a phase transition was again observed. This time, however, the body-centred iron transformed into six-fold symmetry. A possible shear mechanism is shown in figure 9 . Six-fold symmetry can be obtained by shuffling every other (100) plane.

\section{Shear deformation of copper small crystals}

The deformation mechanism is much simpler in face-centred cubic lattice than that in 
body-centred cubic lattice. As an example a face-centred metal, copper, was chosen. $\mathrm{N}$-body embedded function (potential $\mathrm{Cu}-2$ ) was used. A small crystal of rectangular parallelipiped having the faces $(111),(11 \overline{2}),(1 \overline{1} 0),(\overline{1} \overline{1}),(\overline{1} \overline{1} 2)$ and $(\overline{1} 10)$ was created in the computer. The specimen contains 1890 atoms. The periodic boundary condition in [112] was used. The specimen is called specimen $\mathrm{Cu}-1$ hereafter (figure 10a).

On (1T) plane, stepwise displacement of $0.08 d$, where $d$ is the nearest-neighbour distance, was given, then relaxation for 100 cycles, again $0.08 d$ displacement, and relaxation for 100 cycles. The process was repeated. The projections of atomic positions on $(11 \overline{2})$ are given in figures $11 \mathrm{a}$ to $11 \mathrm{c}$. The projections of atoms on (111) are given in figures $12 \mathrm{a}$ to $12 \mathrm{c}$. Dislocations were created on the (111) and moved through and ejected from the crystal. The first dislocation created was a half

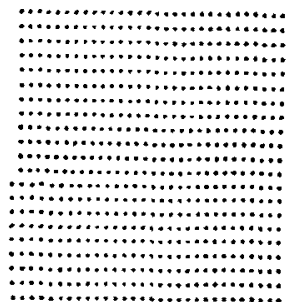

(a)

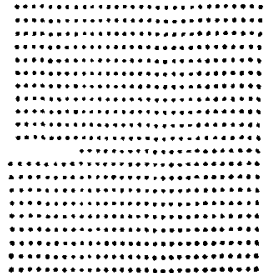

(d)

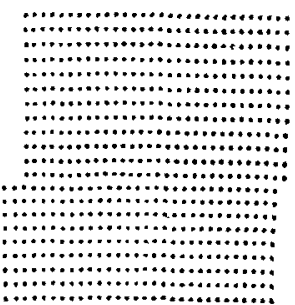

(b)

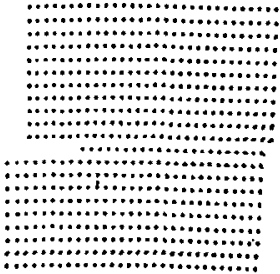

(e)

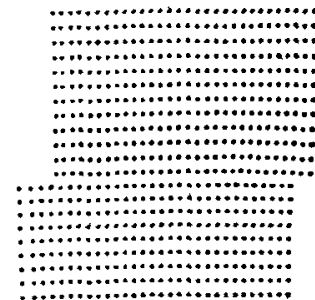

(c)

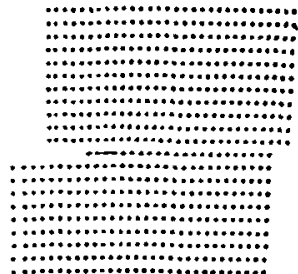

(f)

Figure 11. Projection of atom positions of copper specimen $\mathrm{Cu}-1$ on (112). Creation of dislocations. Step heights are (a) $0-4 d$, (b) $1 \cdot 2 d$ and (c) $2 d$. With a notch, specimen $\mathrm{Cu}-2$, (d) $0-4 d$, (e) $1-2 d$ and (f) $2 d$.

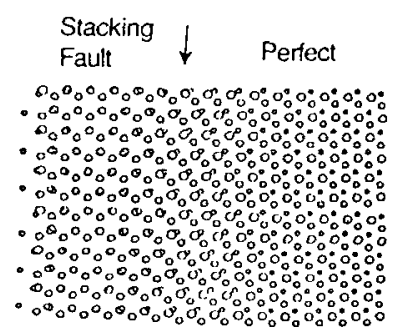

(a)

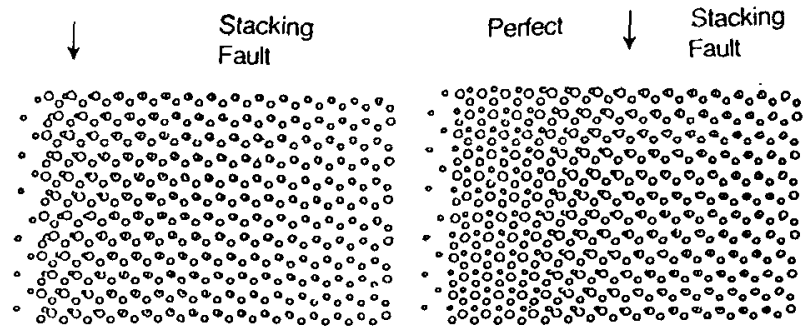

(b) (c)

Figure 12. Projection of copper atoms on (111). Two half-dislocations are connected with a stacking fault. (a) At 3500 time cycles (0.7d step height), (b) at 5000 time cycles (1.0d step height), and (c) at 6000 time cycles (0.7d step height). Arrows show half-dislocations. 


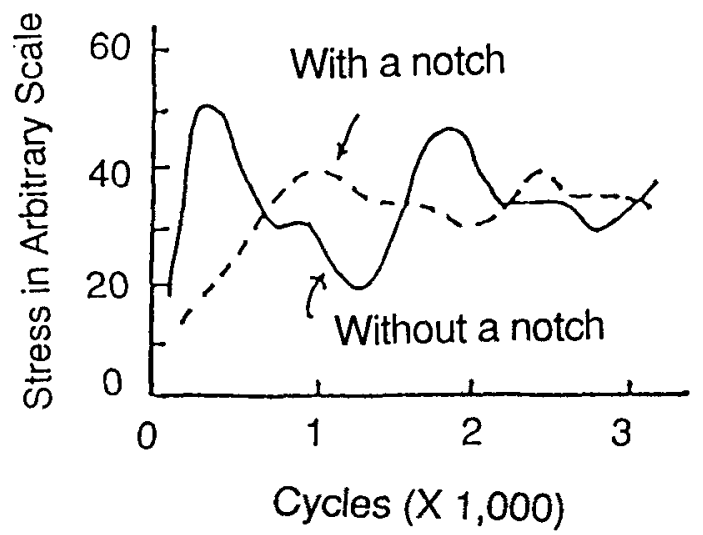

Figure 13. Stress - strain curves of specimen $\mathrm{Cu}-1$ (without a notch) and $\mathrm{Cu}-2$ (with a notch).

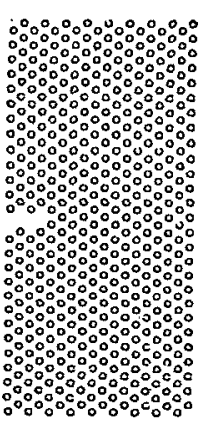

(a)

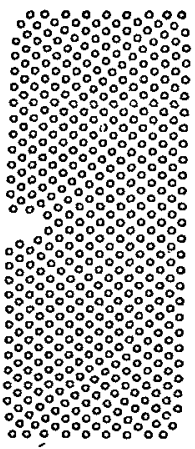

(b)

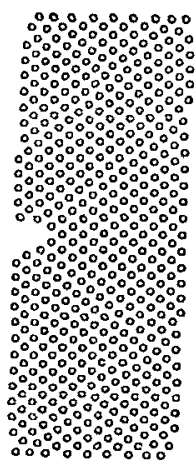

(c)

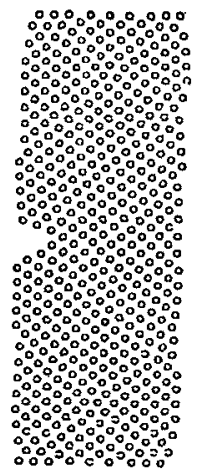

(d)

Figure 14. Specimen Cu-3 (A1 deformation), projection on $x z$ plane: (a) at 300 time cycles (6\% elongation), (b) at 375 cycles ( $7.5 \%$ elongation), (c) at 500 cycles ( $10 \%$ elongation), and (d) at 675 cycles ( $13.5 \%$ elongation).

Heidenreich-Shockley dislocation and the second dislocation was the other half of the Heidenreich-Shockley dislocation and these half-dislocations were connected by the stacking fault. The stress on the surface of $(1 \overline{1} 0)$ was calculated by the sum of the force in the [101] direction on the atom in the two atomic layers on (110).

For a crystal with a notch, similar deformation was applied. A crack was made by taking out one atomic layer on (111). This specimen is called $\mathrm{Cu}-2$ (figure 10b). Dislocations were created from the tip of the notch, slided on (111), and ejected. The projection of atomic positions on (112) are given in figures $11 \mathrm{~d}$ to $11 \mathrm{f}$. The stressstrain curves are shown in figure 13. It was found that the stress to create the first halfdislocation into the crystal was the highest and the stress to create the second half-dislocation was lower, and that the stress to create the second dislocation is a little lower than that for the first one. The stress to create dislocations in the crystal with a notch (specimen $\mathrm{Cu}-2$ ) was lower than that in the crystal without a notch (specimen $\mathrm{Cu}-1)$. The projection of atomic positions on $(11 \overline{2})$ are similar to figures 11a to $11 \mathrm{c}$. Edge dislocations were created on (110). The edge dislocations were split into 
two Heidenreich-Shockley partial dislocations. At the beginning, the first Heidenreich-Shockley partial dislocation was created and the stacking fault was extended to the surface. When the second partial dislocation was created the two partial dislocations were connected by the stacking fault. Figure 12 shows the projections to (111) plane of the atomic positions near the slip plane. The stacking fault is very clearly seen in these figures. The left-hand side of figure $14 a$, the right-hand side of figure $12 b$, and the right-hand side of figure $12 \mathrm{c}$ are typical examples of the stacking faults. Comparing figures $12 \mathrm{c}$ and $12 \mathrm{~d}$, it can be seen that the partial dislocation is advancing in the right hand direction. The area of the stacking fault in figure $12 \mathrm{~d}$ is smaller than that in figure 12e.

In the stress-strain curve (figure 13), very sharp yield stress was found. The stress increased until the first partial dislocation was created. When the second partial dislocation was created, the stress dropped. A stress minimum was the time when the first partial dislocation was pushed out from the crystal of the other surface and the second dislocation was connected with the stacking fault which is extended to the surface of the other side. The heights of peaks are lower as the deformation proceeds.

\section{Tensile deformation of small copper single crystals using $n$-body embedded function}

The $n$-body embedded function proposed by $\mathrm{Oh}$ and Johnson $\mathrm{Cu}-2(1988,1989)$ was used in this section. Two specimens were prepared. Specimen $\mathrm{Cu}-3$ (figure $10 \mathrm{c}$ ) was a rectangular parallelipiped having the faces $(100),(\overline{1} 00),(010),(0 \overline{1} 0),(001)$ and $(00 \overline{1}) . x$, $y$ and $z$ directions were taken in [100], [001] and [110], respectively. The size of specimen $\mathrm{Cu}-4$ is $12 \mathrm{~d} \times 8 a \times 20 d$, where $a$ is the lattice parameter and $d$ is the nearest-neighbour distance, and has a notch in $y$ direction at the centre on (110) (figure 10d). The number of atoms in specimen $\mathrm{Cu}-4$ is 4896 . The surfaces were free and periodic boundary conditions were not used. Using the same interaction potential, the activation energies and binding energies of ad-atoms and ad-atom clusters have been calculated (Doyama 1993) and reasonable results have been obtained.

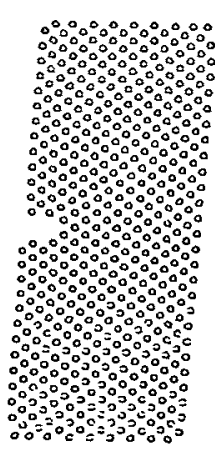

(a)

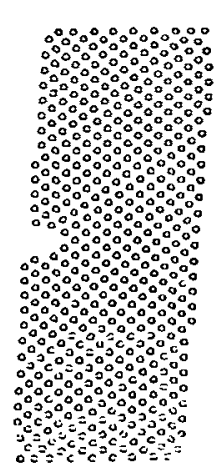

(b)

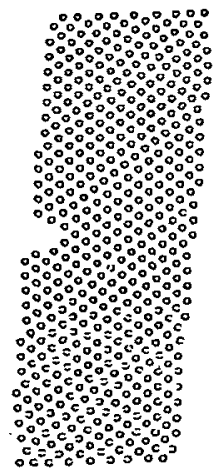

(c)

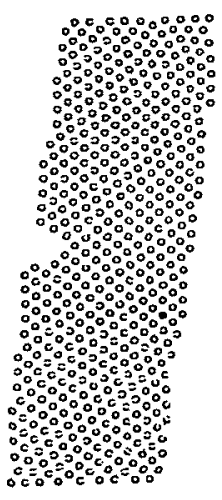

(d)

Figure 15. Specimen $\mathrm{Cu}-3$ (A2 deformation): (a) 300 cycles ( $8 \cdot 4 \%$ elongation), (b) 400 cycles ( $22 \cdot 4 \%$ elongation), (c) 600 cycles ( $33 \cdot 4 \%$ elongation), and (d) 700 cycles (39.2\% elongation). 


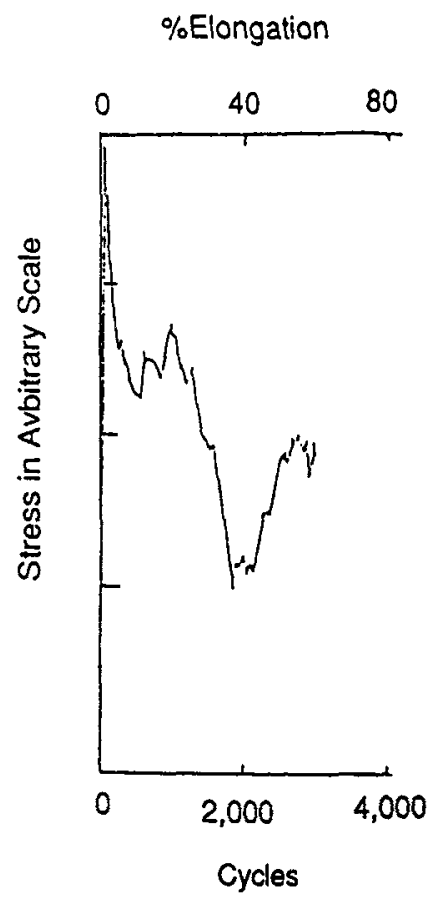

Figure 16. Stress-strain curve of specimen $\mathrm{Cu}-3$ (A2 deformation).

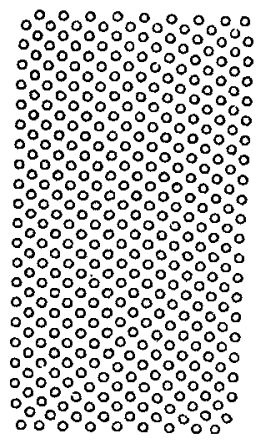

(a)

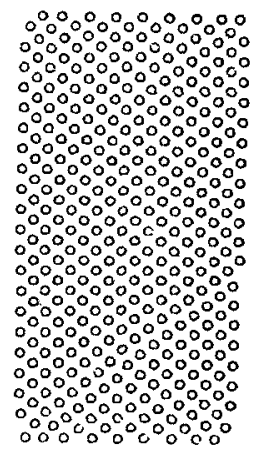

(b)

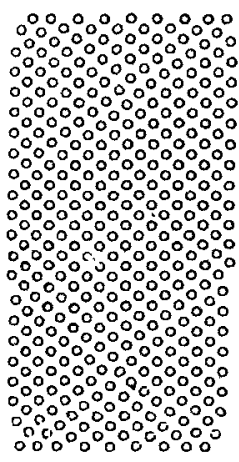

(c)

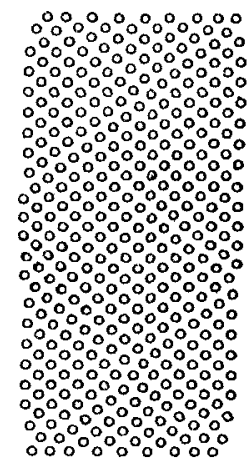

(d)

Figure 17. Specimen $\mathrm{Cu}-3$ (B1 deformation): (a) 450 cycles ( $9 \%$ elongation), (b) 600 cycles ( $12 \%$ elongation), (c) 675 cycles ( $13.5 \%$ elongation), and (d) 750 cycles ( $15 \%$ elongation).

Specimen $\mathrm{Cu}-3$ was pulled $0.5 \%$ in [001] direction ( $\mathrm{Al}$ deformation), all the atoms were deformed uniformly, then all atoms except one layer of each end were relaxed for 25 cycles and again pulled $0.5 \%$ in [001] direction, then all atoms were relaxed for 25 cycles. The atoms in the end layers were relaxed only in $x$ and $y$ directions but not in $z$ direction in the relaxation process. This procedure was repeated. Uniform deformation was given to $\mathrm{Cu}-3$ in [101] direction (A2 deformation): pulled $0.7 \%$ in the [101] 


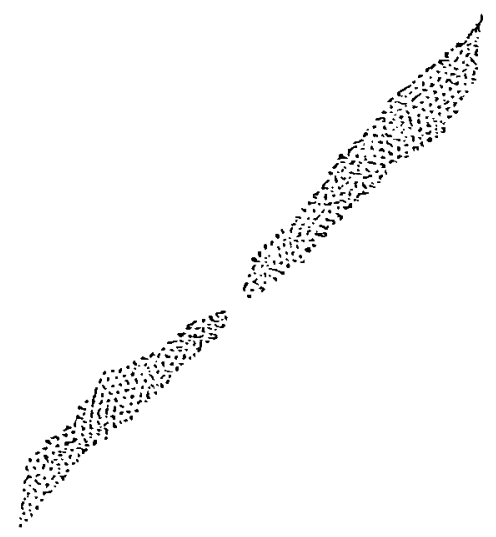

Figure 18. Specimen $\mathrm{Cu}-3$ just after break into two pieces.
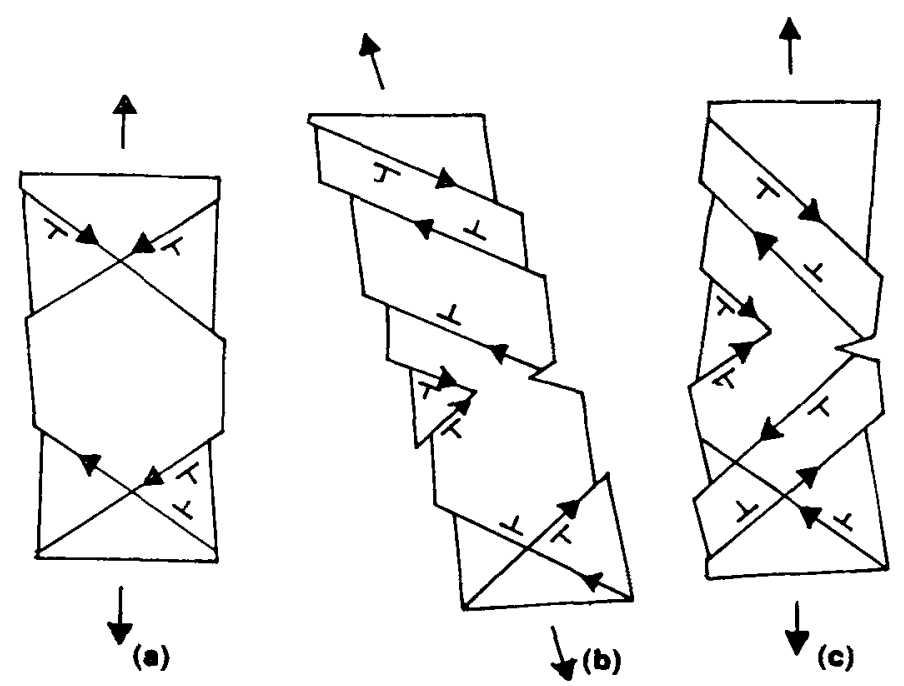

Figure 19. Schematic diagrams of the creation and motion of half-dislocations in (a) specimen $\mathrm{Cu}-3$ (A1 deformation), (b) specimen $\mathrm{Cu}-2$ (A2 deformation), and (c) specimen $\mathrm{Cu}-4$.

direction, all the atoms except one layer of both ends relaxed for 25 cycles, pulled $0 \cdot 7 \%$ in [101] direction and all atoms relaxed for 25 cycles.

Specimen $\mathrm{Cu}-4$ was pulled in [110] direction (G1 deformation). All atoms were moved $0.005 \times\left(x_{i}^{2}+z_{i}^{2}\right)^{1 / 2}$ in [101] direction, then all the atoms except one layer of the both ends were relaxed for 25 cycles, pulled $0.5 \%$ and all atoms relaxed for 25 cycles. The atoms in one layer of the both ends were relaxed in $x$ and $y$ directions. This was repeated. The time step of the molecular dynamics was $5 \times 10^{-15} \mathrm{sec}$.

Atomic positions near the centre in the $y$ direction of specimen $\mathrm{Cu}-3$ (A1 deformation) projected on $x z$ plane (010) are plotted in figures 14(a), (b), (c) and (d), figure 14a is the initial configuration. In figure $14 \mathrm{~b}$, near the tip of the notch, a half-dislocation was created. Two other half-dislocations are seen near the grip. These can be clearly seen by looking at the figures at a small angle from the paper. Atomic positions near 
the centre of $\mathrm{Cu}-3$ (A2 deformation) projected on $x z$ plane (010) are plotted in figure 15. A half-dislocation was created near the tip of the crack, and two halfdislocations were created near the grip. This is more clearly seen in figure $15 \mathrm{c}$. In figure 16 the stress-strain curve of specimen $\mathrm{Cu}-3$ (A2 deformation) is plotted. A very sharp yield point was found. Atomic positions near the centre of specimen $\mathrm{Cu}-4$ projected to $x z$ plane are plotted in figure 17 . In figure $17 \mathrm{a}$ a half-dislocation is seen near the grip. More dislocations are seen in figures $16 \mathrm{c}$ and $16 \mathrm{~d}$. The projection on $x z$ plane just after fracture is plotted in figure 18 .

It was found that a notch is normally a source of dislocations due to stress concentration. When the notch thickness was small however, (in the case of figure 17), the effect of the notch was not large. The interaction across the notch still existed. [211] (111) and [112] (111) half-dislocations were created leaving stacking faults. Schematic diagrams are given in figures 19 (a), (b) and (c) for specimen $\mathrm{Cu}-3$ (A1 deformation), specimen $\mathrm{Cu}-2$ (A2 deformation) and specimen $\mathrm{Cu}-4$, respectively. The initial stage of deformation was surely caused by the creation and motion of dislocations. In the middle stage, dislocations on different slip planes cross each other. The work hardening occurs due to the cutting of dislocations. In the final stage, in the case of copper, crystalline state with many dislocations and the inhomogeneous is normal.

\section{Acknowledgement}

The research was supported by a Grant-in-Aid by the Ministry of Education, Science and Culture under Priority Area "Crystal Growth Mechanism in Atomic Scale".

\section{References}

Doyama M 1993 Computer aided innovation of new materials II (eds) M Doyama, J Kihara, M Tanaka and R Yamamoto (Amsterdam: North-Holland) pp 587-590

Doyama M and Yamamoto R 1986 Computational mechanics 86 (Springer Verlag) part III, pp 203-208

Oh D J and Johnson R A 1988 J. Mater. Res. 3471

Oh D J and Johnson R A 1989 Atomistic simulation of materials (eds) V Vitek and D J Srolovitz (New York: Plenum Press) pp 233-238

Pak H M and Doyama M 1969 J. Fac. Engg. B30 111 